\title{
Associação entre o estilo de vida dos pais e a composição corporal dos filhos adolescentes
}

\author{
Association of parental lifestyle with body composition of their adolescent children
}

\author{
Edio Luiz Petroski', Andreia Pelegrini²
}

\section{RESUMO}

Objetivo: Verificar a associação entre o estilo de vida dos pais e a composição corporal de seus filhos adolescentes.

Métodos: Participaram do estudo 120 indivíduos, sendo 40 adolescentes de 14 a 17 anos do sexo masculino e seus respectivos pais $(n=80)$. Os adolescentes foram divididos em dois grupos de acordo com o percentual de gordura $(\% \mathrm{G})$ : baixo $(\% \mathrm{G}<11 \%)$ e alto $(\% \mathrm{G}>20 \%)$. Mensuraram-se o peso, a estatura e as dobras cutâneas (tríceps e subescapular) e estimou-se o \% G dos adolescentes por meio da equação de Lohman. O questionário "estilo de vida fantástico" foi respondido pelos pais. Para a análise estatística, utilizou-se o teste exato de Fischer, considerando-se significante $p<0,05$.

Resultados: A prevalência de excesso de peso foi duas vezes maior nos pais de adolescentes com \% G alto em comparação aos pais daqueles com \% baixo (42 e 25\%; 75 e $15 \%$, para mães e pais respectivamente). O estilo de vida dos pais dos adolescentes com $\% \mathrm{G}$ baixo foi significativamente melhor que os pais dos adolescentes com \% G alto.

Conclusões: $\mathrm{O}$ estilo de vida dos pais esteve associado à composição corporal dos filhos com $\% \mathrm{G}$ abaixo ou acima do ideal.

Palavras-chave: adiposidade; adolescente; exercício; composição corporal; estilo de vida; estado nutricional.

\section{ABSTRACT}

Objective: Investigate the association of parental lifestyle with body composition of their adolescent offspring.

Methods: The study included 120 individuals: 40 male adolescents aged between 14 and 17 years and their parents $(n=80)$. The adolescents were divided into two groups according to the percentage of body fat (\%BF): low $(\% \mathrm{BF}<11 \%)$ or high $(\% \mathrm{BF}>20 \%)$. Body weight, stature and triceps and subscapular skinfolds were measured, and the Lohman equation was used to estimate $\% \mathrm{BF}$ of the adolescents. The parents answered the FANTASTIC lifestyle questionnaire. Statistical analyses included Fisher's exact test, being significant $p<0.05$.

Results: The prevalence of overweight among both parents of adolescents with high \% BF was twice the prevalence observed among parents of adolescents with low \% BF (42 and $25 \%$; 75 and $15 \%$, for mothers and fathers, respectively). The lifestyle of the parents of adolescents with low $\% \mathrm{BF}$ was significantly better than the parents of adolescents with high $\% \mathrm{BF}$.

Conclusions: Lifestyle of parents is associated with body composition of adolescent offspring with high and low $\% \mathrm{BF}$.

Key-words: adiposity; adolescent; exercise; body composition; life style; nutritional status.
Instituição: Universidade Federal de Santa Catarina (UFSC), Florianópolis, $\mathrm{SC}$, Brasil

'Doutor em Educação Física pela Universidade Federal de Santa Maria, professor do Programa de Pós-Graduação em Educação Física da UFSC, líder do Grupo de Pesquisa em Cineantropometria e Desempenho Humano (NuCIDH), Florianópolis, SC, Brasil

2Doutoranda do Programa de Pós-Graduação em Educação Física da UFSC, membro do Grupo de Pesquisa NuCIDH, bolsista da Coordenação de Aperfeiçoamento de Pessoal de Nível Superior, Florianópolis, SC, Brasil
Endereço para correspondência:

Edio Luiz Petroski

$\mathrm{NuCIDH/Centros} \mathrm{de} \mathrm{Desportos/UFSC}$

Campus Universitário, Caixa Postal 476 - Trindade

CEP 88040-900 - Florianópolis/SC

E-mail: petroski@cds.ufsc.br

Recebido em: 9/5/08

Aprovado em: 20/8/08 


\section{Introdução}

O estilo de vida é caracterizado como um padrão de comportamento que pode ter profundo efeito na saúde dos seres humanos e está relacionado a aspectos que refletem as atitudes, os valores e as oportunidades na vida das pessoas ${ }^{(1)}$.

Nos últimos anos, mudanças no estilo de vida de indivíduos de todas as idades têm sido observadas, favorecendo o aumento dos fatores de risco à saúde, principalmente devido ao aumento da prevalência de sobrepeso e obesidade em todas as faixas etárias. Nesse sentido, a relação entre sedentarismo e excesso de peso corporal está diretamente associada ao risco de doenças cardiovasculares, metabólicas e psíquicas ${ }^{(2)}$, tornando a obesidade uma das principais ameaças do mundo industrializado ${ }^{(3)}$.

Se, por um lado, o sedentarismo é um agravante para o aumento da prevalência de sobrepeso e obesidade, por outro, o exercício físico é considerado um fator de proteção na prevenção primária e secundária das doenças cardiovasculares e no controle da hipertensão $\operatorname{arterial}^{(4)}$, pois auxilia no controle das dislipidemias, na diminuição do nível de glicemia, na prevenção da osteoporose ${ }^{(5,6)} \mathrm{e}$, também, no tratamento da obesidade $^{(7)}$. A literatura tem recomendado, no mínimo, 30 minutos de exercício físico aeróbio moderado cinco vezes por semana ou exercício vigoroso durante 20 minutos, três vezes por semana, como suficiente para promover e manter a saúde. Além disso, sugere-se a realização de exercícios de contrarresistência nos principais grupamentos musculares, pelo menos dois dias da semana, para manter e/ou aumentar a força e a resistência musculares ${ }^{(8)}$.

Embora a maioria das doenças associadas ao sedentarismo somente se manifeste na vida adulta, é cada vez mais evidente que seu desenvolvimento se inicia na infância e adolescência, o que pode ser explicado, em parte, pela influência dos pais na adoção de hábitos sedentários pelos filhos. Dessa forma, o estímulo à prática de atividades físicas em idades mais jovens deve ser uma prioridade em saúde pública, uma vez que o envolvimento em atividades físicas diárias exerce papel significativo na promoção da saúde e na prevenção de doenças hipocinéticas ${ }^{(5)}$. Tendo isso em mente, o propósito do presente estudo foi verificar a associação entre o estilo de vida dos pais e a composição corporal dos filhos.

\section{Métodos}

No desenvolvimento deste estudo, utilizaram-se dados coletados em um estudo do tipo survey, intitulado "Rela- ção entre nível de atividade física e hábitos alimentares de adolescentes e estilo de vida dos pais"(9), caracterizado como pesquisa do tipo descritiva correlacional. Após a aprovação do Comitê de Ética em Pesquisa e a autorização dos pais por meio da assinatura do termo de consentimento livre e esclarecido, a coleta de dados foi iniciada.

A amostra foi selecionada de forma intencional, pois os adolescentes deveriam atender aos seguintes critérios de inclusão: pertencer às classes econômicas $\mathrm{A}$ e $\mathrm{B}^{(10)}$; ser da raça branca; não ser fumante e possuir percentual de gordura (\%G) menor que $11 \%$ e maior que $20 \%$ do peso corporal ${ }^{(11)}$. Posteriormente, para compor a amostra, foram selecionados, de forma aleatória, 120 sujeitos, sendo eles 40 adolescentes de 14 a 16 anos do sexo masculino e seus respectivos pais ( $\mathrm{n}=80 ; 40$ pais e 40 mães). Os adolescentes constituíram dois grupos de 20 sujeitos com $\% \mathrm{G}$ baixo $(<11 \%)$ e $\% \mathrm{G}$ alto $(>20 \%)$, classificados como abaixo e acima do ideal, respectivamente.

As medidas de peso corporal, estatura ${ }^{(12)}$ e dobras cutâneas das regiões tricipital e subescapular ${ }^{(13)}$ foram mensuradas por um único avaliador treinado. $\mathrm{O}$ peso corporal foi medido com uma balança digital e a estatura com fita métrica fixada na parede. As espessuras das dobras cutâneas foram aferidas com adipômetro da marca Lange. O percentual de gordura $(\% \mathrm{G})$ dos adolescentes foi classificado de acordo com os pontos de corte sugeridos por Lohman ${ }^{(14)}$. As variáveis antropométricas (massa corporal, estatura) e o estilo de vida dos pais foram coletados durante uma visita à casa dos adolescentes. Foram considerados com sobrepeso os pais com índice de massa corpórea (IMC) entre 25 e $29,9 \mathrm{~kg} / \mathrm{m}^{2}$ e obesos, aqueles com IMC superior a $30 \mathrm{~kg} / \mathrm{m}^{2(15)}$.

Para determinar o estilo de vida, utilizou-se o questionário autoadministrado "Estilo de vida fantástico", o qual faz parte de um conjunto de procedimentos que constituem a bateria de testes denominada Plano Canadense de Avaliação da Atividade Física, Aptidão e Estilo de Vida ${ }^{(16)}$. O questionário possui 25 questões divididas em nove domínios: 1) família e amigos; 2) atividade física; 3) nutrição; 4) tabaco e tóxicos; 5) álcool; 6) sono, cinto de segurança, estresse e sexo seguro; 7) tipo de comportamento; 8) introspecção e 9) trabalho. Com relação à padronização de termos, empregou-se "pais" para representar os sujeitos paternos, "mães" para representar os maternos e "ambos os pais" para fazer referência aos dois termos citados anteriormente.

A normalidade dos dados foi verificada por meio do teste Shapiro Wilk. Para caracterizar as variáveis analisadas, 
recorreu-se à estatística descritiva (média, desvio padrão, valores máximos e mínimos). Na comparação entre o estilo de vida dos pais e a gordura corporal dos filhos, utilizou-se o teste exato de Fischer. O nível de significância foi fixado em $5 \%$. Todos os procedimentos foram realizados no pacote computacional SPSS versão 11.0.

\section{Resultados}

$\mathrm{Na}$ Tabela 1 estão relacionados os indicadores antropométricos dos adolescentes de acordo com o percentual de gordura. Os resultados demonstraram que os adolescentes com $\% \mathrm{G}$ alto apresentaram-se mais pesados, com maiores valores de IMC e maior $\% \mathrm{G}$ em relação àqueles com $\% \mathrm{G}$ baixo $(p<0,05)$, o que já era esperado, devido à metodologia empregada no estudo.

Foi possível identificar que os pais dos adolescentes com $\% \mathrm{G}$ alto eram mais pesados e mais altos que os pais dos adolescentes com $\% \mathrm{G}$ baixo $(p<0,05$, Tabela 2). Da mesma forma, as mães dos adolescentes com $\% \mathrm{G}$ alto eram mais pesadas, mais altas e apresentaram maiores valores de $\operatorname{IMC}(p<0,05)$ em relação às mães dos adolescentes com $\% \mathrm{G}$ baixo.
O Gráfico 1 demonstra que os pais dos adolescentes com $\% \mathrm{G}$ alto apresentaram prevalência de excesso de peso (sobrepeso+obesidade) duas vezes maior que os pais dos adolescentes com \% G baixo. Em relação às mães, a maioria foi classificada com IMC normal. Todavia, o sobrepeso foi quase duas vezes maior nas mães dos adolescentes com $\% \mathrm{G}$ alto do que nas mães daqueles com $\% \mathrm{G}$ baixo.

Os pais dos adolescentes com $\% \mathrm{G}$ alto demonstraram variação do estilo de vida de regular a muito bom e os pais dos adolescentes com \%G baixo, de regular a excelente. Por outro lado, o estilo de vida das mães dos adolescentes com \% G alto variou de bom a excelente e, entre as mães dos adolescentes com \% G baixo, de regular a muito bom (Gráfico 2).

De acordo com os diferentes padrões de $\% \mathrm{G}$ dos filhos (Tabela 3), observa-se que o estilo de vida dos pais dos adolescentes com $\% \mathrm{G}$ baixo foi melhor do que daqueles com $\% \mathrm{G}$ alto $(p<0,05)$, enquanto as mães apresentaram estilo de vida similar.

Para uma análise melhor da classificação dos estilos de vida, esses foram agrupados em excelente/muito bom e bom/regular (Tabela 3). Os resultados demonstraram associações entre o estilo de vida de ambos os pais e a com-

Tabela 1 - Indicadores antropométricos dos adolescentes de acordo com a gordura corporal

\begin{tabular}{lccc}
\hline & \multicolumn{3}{c}{ Filhos } \\
\cline { 2 - 4 } & Total & \%G baixo & \%G alto \\
\hline Massa corpórea $(\mathrm{kg})$ & $62,5 \pm 15,3$ & $52,8 \pm 10,7$ & $72,6 \pm 12,4^{*}$ \\
Estatura $(\mathrm{cm})$ & $169,6 \pm 9,9$ & $166,8 \pm 9,0$ & $172,4 \pm 10,2$ \\
IMC $\left(\mathrm{kg} / \mathrm{m}^{2}\right)$ & $21,5 \pm 3,9$ & $18,6 \pm 2,1$ & $24,3 \pm 3,0^{*}$ \\
$\%$ de gordura & $16,8 \pm 8,4$ & $9,1 \pm 1,2$ & $24,6 \pm 4,2^{*}$ \\
\hline
\end{tabular}

IMC: índice de massa corpórea; \%G: percentual de gordura; *diferença entre os adolescentes com \%G baixo e \%G alto: $p<0,05$.

Tabela 2 - Indicadores antropométricos dos pais de acordo com a classificação de gordura corporal dos filhos

\begin{tabular}{lcccc}
\hline & \multicolumn{2}{c}{ Pai } & \%G baixo & MG alto \\
\cline { 2 - 5 } & \%G baixo & \%G alto & $55,9 \pm 7,4$ & $65,5 \pm 8,4^{*}$ \\
\hline Massa corpórea $(\mathrm{kg})$ & $75,8 \pm 9,8$ & $83,8 \pm 9,2^{*}$ & $1,6 \pm 0,03$ & $1,6 \pm 0,1^{*}$ \\
Estatura $(\mathrm{m})$ & $1,7 \pm 0,04$ & $1,7 \pm 0,1^{*}$ & $22,8 \pm 2,6$ & $25,0 \pm 2,9^{*}$ \\
IMC $\left(\mathrm{kg} / \mathrm{m}^{2}\right)$ & $26,0 \pm 3,9$ & $27,3 \pm 2,3$ &
\end{tabular}

\%G: percentual de gordura; IMC: índice de massa corpórea; *diferença dos indicadores antropométricos dos pais e mães em relação à gordura corporal dos filhos: $p<0,05$.

Tabela 3 - Associação entre a classificação do estilo de vida de ambos os pais e o \%G dos filhos

\begin{tabular}{llcc}
\hline & \multirow{2}{*}{ Estilo de vida } & \multicolumn{2}{c}{ Adolescentes } \\
\cline { 3 - 4 } & Excelente/muito bom & \%G baixo & \%G alto \\
\hline Pais & Bom/regular & 55,6 & $15,8^{*}$ \\
\multirow{2}{*}{ Mães } & Excelente/muito bom & 44,4 & $84,2^{*}$ \\
& Bom/regular & 44,4 & $80,0^{*}$ \\
& & 55,6 & $20,0^{*}$ \\
\hline
\end{tabular}

$\%$ G: percentual de gordura; ${ }^{*} p<0,05$. 
posição corporal dos filhos $(p<0,05)$. Enquanto os pais com estilo de vida excelente/muito bom possuíam filhos com menor acúmulo de gordura corporal, as mães com melhor estilo de vida possuíam filhos com \%G acima dos valores recomendados para a saúde.

\section{Discussão}

De acordo com os resultados encontrados, os pais dos adolescentes com $\% \mathrm{G}$ alto eram mais pesados e mais altos que os pais dos adolescentes com \%G baixo. Já as mães dos adolescentes com $\% \mathrm{G}$ alto, além de mais pesadas, eram mais altas e apresentaram valores de IMC maiores do que as mães dos jovens com $\% \mathrm{G}$ baixo.

Evidências nacionais têm demonstrado associação entre o IMC dos pais e a gordura corporal dos filhos ${ }^{(17-19)}$. Em Capão da Canoa, Rio Grande do Sul, verificou-se que a prevalência de sobrepeso e obesidade em adolescentes aumentou de acordo com o estado nutricional dos pais. Além disso, houve associação linear significativa, de modo que os adolescentes com pelo menos um dos pais acima do peso apresentaram cerca de $50 \%$ a mais de risco de sobrepeso e obesidade. Aqueles com ambos os pais acima do peso mostraram o dobro de risco de sobrepeso e obesidade ${ }^{(20)}$.

Estudos recentes sugerem que o IMC dos pais cujos filhos apresentam excesso de peso é maior do que o IMC de pais de crianças com peso normal ${ }^{(20)}$, sendo a chance de excesso de peso três a sete vezes maior em crianças e adolescentes com pais e mães obesos, comparada àquelas cujos pais apresentam peso normal $^{(17,21)}$. Na Austrália, verificou-se que o IMC dos filhos foi significativamente predito pelo IMC de ambos os pais ${ }^{(22)}$.

O peso corporal da mãe pode ser considerado fator preditor no acúmulo de gordura corporal dos filhos ${ }^{(23)}$. A associação da obesidade na infância e na adolescência com valores mais altos de IMC dos pais é provavelmente influenciada pela herança genética, adoção de hábitos sedentários, inadequação alimentar e/ou fatores relacionados às condições de vida ${ }^{(24)}$.

No que diz respeito ao estilo de vida, os achados do presente estudo demonstraram que os pais dos adolescentes com \% G baixo possuíam estilo de vida mais favorável à saúde. A literatura tem demonstrado que o estilo de vida ativo inicia-se na infância e adolescência ${ }^{(25)}$ e que indivíduos envolvidos em atividade física na adolescência apresentam maior probabilidade de serem suficientemente ativos na idade adulta ${ }^{(26)}$.

Em pesquisa realizada com pais, mães e filhas adolescentes $^{(27)}$, verificou-se que os adultos tendem a favorecer estratégias diferentes ao encorajar as filhas a serem ativas:

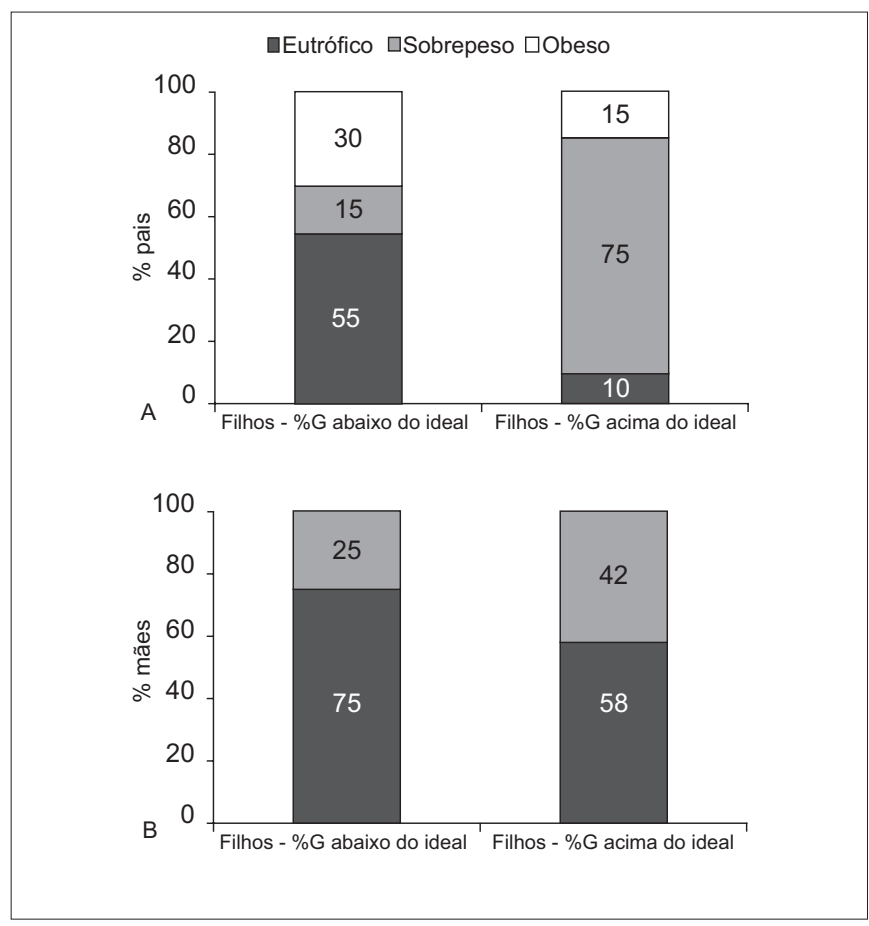

Gráfico 1 - Estado nutricional dos pais e mães em relação ao percentual de gordura $(\% \mathrm{G})$ dos filhos.

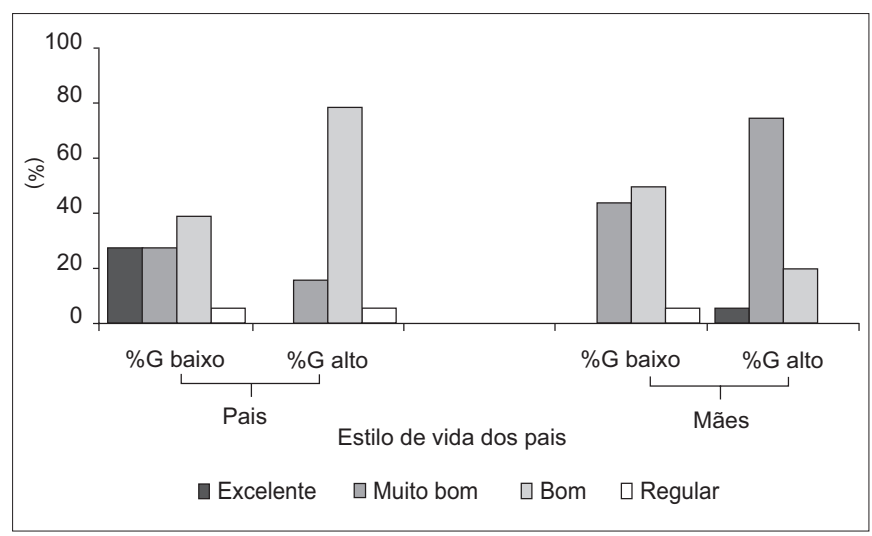

Gráfico 2 - Classificações do estilo de vida de ambos os pais de acordo com o percentual de gordura $(\% \mathrm{G})$ dos filhos.

o pai utiliza o seu comportamento como exemplo e a mãe atua como apoiadora. Portanto, a aderência dos adolescentes a práticas regulares de atividade física está associada à conscientização e ao incentivo de ambos os pais na adoção de um estilo de vida saudável ${ }^{(28)}$.

Há evidência de que basta um dos pais ser ativo para o adolescente ser ativo ${ }^{(28)}$. Mães ativas possuem duas vezes mais chances de terem filhos ativos e, quando ambos os pais demonstram esse comportamento, a chance aumenta em cinco vezes ${ }^{(29)}$. Portanto, os pais são influência forte na prática de exercício físico dos filhos, ou ainda, as atitudes dos pais 
estimulam, de maneira geral, a obtenção de comportamentos saudáveis por parte de crianças e adolescentes ${ }^{(28,30)}$.

O estudo sugere uma associação positiva do estilo de vida dos pais à composição corporal dos filhos com $\% \mathrm{G}$ abaixo ou acima do ideal. Embora não tenha sido foco de análise neste estudo, observou-se que os adolescentes com \%G baixo eram mais ativos fisicamente (quando mensurados com acelerômetro Tritrac, durante uma semana habitual) do que aqueles com $\% \mathrm{G}$ alto ${ }^{(9)}$.

\section{Referências bibliográficas}

1. World Health Organization. The World Health report 1998 - life in the $21^{\text {st }}$ century: a vision for all. Geneva: World Health Organization; 1998.

2. Campbell KL, Crocker PR, McKenzie DC. Field evaluation of energy expenditure in women using Tritrac accelerometers. Med Sci Sports Exerc 2002;34:1667-74.

3. World Health Organization. The World Health report 2002 - reducing risks, promoting healthy life. Geneva: World Health Organization; 2002.

4. Paffenbarger RS Jr, Hyde RT, Wing AL, Lee IM, Jung DL, Kampert JB. The association of changes in physical-activity level and other lifestyle characteristics with mortality among men. N Engl J Med 1993;328:538-45.

5. Strong WB, Malina RM, Blimkie CJ, Daniels SR, Dishman RK, Gutin B et al. Evidence based physical activity for school-age youth. J Pediatr 2005;146:732-7.

6. Yancey AK, Fielding JE, Flores GR, Sallis JF, McCarthy WJ, Breslow L. Creating a robust public health infrastructure for physical activity promotion. Am J Prev Med 2007;32:68-78.

7. Blair SN, Horton E, Leon AS, Lee IM, Drinkwater BL, Dishman RK et al. Physical activity, nutrition, and chronic disease. Med Sci Sports Exerc 1996;28:335-49.

8. Haskell WL, Lee IM, Pate RR, Powell KE, Blair SN, Franklin BA et al. Physical activity and public health: updated recommendation for adults from the American College of Sports Medicine and the American Heart Association. Med Sci Sports Exerc 2007;39:1423-34.

9. Ilha PM. Relação entre nível de atividade física e hábitos alimentares de adolescentes e estilo de vida dos pais [dissertação de Mestrado]. Florianópolis (SC): UFSC; 2004.

10. Associação Brasileira de Estudos Populacionais-ABEP [homepage on the Internet]. Critério de Classificação Econômica Brasil (2000). [cited 2008 Feb 12]. Available from: http://www.abep.org/codigosguias/ABEP_CCEB.pdf

11. Lohman TG. Applicability of body composition techniques and constants for children and youths. Exerc Sport Sci Rev 1986;14:325-57.

12. Alvarez BR, Pavan AL. Alturas e comprimentos. In: Petroski EL, editor. Antropometria: técnicas e padronizações. $4^{a}$ ed. Blumenau: Nova Letra; 2007. p. 31-44

13. Benedetti TR, Pinho RA, Ramos VM. Dobras cutâneas. In: Petroski EL, editor. Antropometria: técnicas e padronizações. $4^{\mathrm{a}}$ ed. Blumenau: Nova Letra; 2007. p. $45-56$

14. Lohman TG. The use of skinfold to estimate body fatness on children and youth. JOPERD 1987:58:98-102.

15. [No authors listed]. Physical status: the use and interpretation of anthropometry: report of a WHO expert committe. World Health Organ Tech Rep Ser 1995:854:1-452.
As principais limitações da pesquisa referem-se ao tamanho da amostra e a características específicas dos adolescentes (classe econômica elevada, \% G abaixo e acima do ideal). Dessa forma, os resultados devem ser interpretados com cautela. Entretanto, conclui-se que o estilo de vida dos pais esteve associado à composição corporal dos filhos com $\% \mathrm{G}$ abaixo ou acima do recomendado. Nesse sentido, pode-se entender a importância do estilo de vida dos pais no planejamento de programas de atividades físicas para adolescentes.

16. Rodriguez-Añez CR, Reis RS, Petroski EL. Versão brasileira do questionário de estilo de vida: tradução e validação para adultos. Arq Bras Cardiol. In press 2008.

17. Guimarães LV, Barros MB, Martins MS, Duarte EC. Fatores associados ao sobrepeso em escolares. Rev Nutr 2006;19:5-17.

18. Ramos AM, Barros Filho AA. Prevalência da obesidade em adolescentes de Bragança Paulista e sua relação com a obesidade dos pais. Arq Bras Endocrinol Metab 2003;47:663-8.

19. Suñé FR, Dias-da-Costa JS, Olinto MT, Pattussi MP. Prevalence of overweight and obesity and associated factors among schoolchildren in a southern Brazilian city. Cad Saude Publica 2007;23:1361-71.

20. Giugliano R, Carneiro EC. Fatores associados à obesidade em escolares. J Pediatr (Rio J) 2004;80:17-22.

21. Isabela da Costa R, Taddei JA, Colugnatti F. Obesity among children attending elementary public schools in São Paulo, Brazil: a case-control study. Public Health Nutr 2003;6:659-63.

22. Burke V, Beilin LJ, Dunbar D. Family lifestyle and parental body mass index as predictors of body mass index in Australian children: a longitudinal study. Int J Obes Relat Metab Disord 2001;25:147-57.

23. Field AE, Austin SB, Gillman MW, Rosner B, Rockett HR, Colditz GA. Snack food intake does not predict weight change among children and adolescents. Int J Obes Relat Metab Disord 2004;28:1210-6.

24. Whitaker RC, Wright JA, Pepe MS, Seidel KD, Dietz WH. Predicting obesity in young adulthood from childhood and parental obesity. N Engl J Med 1997;337:869-73.

25. Pakpreo PM, Ryan MD, Auinger MS, Aten M. The association between parental lifestyle behaviors and adolescent knowledge, attitudes, intentions, and nutritional and physical activity behaviors. J Adolesc Health 2004;34:129-30.

26. Azevedo MR, Araújo CL, Silva MC, Hallal, PC. Tracking of physical activity from adolescence to adulthood: a population-based study. Rev Saude Publica 2007;41:69-75.

27. Davison KK, Cutting TM, Birch LL. Parents' activity-related parenting practices predict girls' physical activity. Med Sci Sports Exerc 2003;35:1589-95.

28. Trost SG, Owen N, Bauman AE, Sallis JF, Brown W. Correlates of adults participation in physical activity: review and update. Med Sci Sports Exerc 2002;34:1996-2001.

29. Moore LL, Lombardi DA, White MJ, Campbell JL, Oliveria SA, Ellison RC. Influence of parents' physical activity levels on activity levels of young children. J Pediatr 1991;118:215-9.

30. Fulton JE, Mâsse LC, Watson KB, Shisler JL, Caspersen CJ. Effect of mediating variables on the association between physical activity of parent and child. Med Sci Sports Exerc 2002;34(Suppl 1):S141. 